\title{
Edar/Eda interactions regulate enamel knot formation in tooth
}

\section{morphogenesis}

\author{
Abigail S. Tucker ${ }^{1}$, Denis J. Headon ${ }^{2}$, Pascal Schneider ${ }^{3}$, Betsy M. Ferguson ${ }^{4}$, Paul Overbeek ${ }^{2}$, \\ Jurg Tschopp ${ }^{3}$ and Paul T. Sharpe ${ }^{5, *}$ \\ ${ }^{1} \mathrm{MRC}$ Centre for Developmental Neurobiology, $4^{\text {th }}$ floor New Hunt's House, King's College, Guy's Hospital, London Bridge, \\ London SE1 1UL, UK \\ 2Department of Molecular and Cellular Biology, Baylor College of Medicine, Houston, Texas 77030, USA \\ 3 Institute of Biochemistry, University of Lausanne, Ch. des Boveresses 155, CH-1066 Epalinges, Switzerland \\ ${ }^{4}$ Department of Molecular and Medical Genetics, Oregon Health Sciences University, 318 SW Sam Jackson Park Road, Portland, \\ Oregon 97201, USA \\ 5Department of Craniofacial Development, GKT Dental Institute, King's College, Guy's Hospital, London Bridge, London SE1 9RT, \\ UK \\ *Author for correspondence (e-mail: paul.sharpe@kcl.ac.uk)
}

Accepted 9 August; published on WWW 9 October 2000

\section{SUMMARY}

tabby and downless mutant mice have apparently identical defects in teeth, hair and sweat glands. Recently, genes responsible for these spontaneous mutations have been identified. downless $(D l)$ encodes Edar, a novel member of the tumour necrosis factor (TNF) receptor family, containing the characteristic extracellular cysteine rich fold, a single transmembrane region and a death homology domain close to the $\mathrm{C}$ terminus. tabby $(\mathrm{Ta})$ encodes ectodysplasin-A (Eda) a type II membrane protein of the TNF ligand family containing an internal collagen-like domain. As predicted by the similarity in adult mutant phenotype and the structure of the proteins, we demonstrate that Eda and Edar specifically interact in vitro.

We have compared the expression pattern of $\mathrm{Dl}$ and $\mathrm{Ta}$ in mouse development, taking the tooth as our model system, and find that they are not expressed in adjacent cells as would have been expected. Teeth develop by a well recorded series of epithelial-mesenchymal interactions, similar to those in hair follicle and sweat gland development, the structures found to be defective in tabby and downless mice. We have analysed the downless mutant teeth in detail, and have traced the defect in cusp morphology back to initial defects in the structure of the tooth enamel knot at E13. Significantly, the defect is distinct from that of the tabby mutant. In the tabby mutant, there is a recognisable but small enamel knot, whereas in the downless mutant the knot is absent, but enamel knot cells are organised into a different shape, the enamel rope, showing altered expression of signalling factors (Shh, Fgf4, Bmp4 and Wnt10b). By adding a soluble form of Edar to tooth germs, we were able to mimic the tabby enamel knot phenotype, demonstrating the involvement of endogenous Eda in tooth development. We could not, however, reproduce the downless phenotype, suggesting the existence of yet another ligand or receptor, or of ligand-independent activation mechanisms for Edar. Changes in the structure of the enamel knot signalling centre in downless tooth germs provide functional data directly linking the enamel knot with tooth cusp morphogenesis. We also show that the Lef1 pathway, thought to be involved in these mutants, functions independently in a parallel pathway.

Key words: Tooth, Enamel knot, Downless, Tabby, EDAR, EDA

\section{INTRODUCTION}

The tabby mutant was the first sex-linked gene discovered in the mouse (Falconer, 1952), the phenotype of the analogous human disorder having been described in 1875 by Charles Darwin (1875). The tabby gene ( $T a)$ is analogous to the gene ED1 (previously EDA) in humans, which is responsible for X-linked hypohidrotic (anhydrotic) ectodermal dysplasia (XLHED; Christ-Siemens-Touraine syndrome) (Kere et al., 1996). $\mathrm{Ta}$ is $94 \%$ identical to a recently identified second isoform of the ED1 protein. This isoform is predicted to encode a 391 amino acid type II transmembrane protein with a short collagenous domain, and has similarities to proteins of the tumour necrosis factor (TNF) family. Mutations in this isoform were detected in $95 \%$ of families with XLHED (Bayes et al, 1998: Monreal et al., 1998).

The tabby phenotype is indistinguishable from that seen for mutations in downless, another spontaneous mouse mutation identified in the late 1950s on the A/H strain (Philips, 1960). tabby and downless mice have abnormally shaped or absent 
teeth (Grüneberg, 1965), missing sweat glands and absence of some hair types (Sundberg, 1994). The downless gene $(D l)$ has recently been identified as encoding a novel TNF receptor, which is mutated in the two known strains of downless mutants (Headon and Overbeek, 1999). Mutations in the human homologue of mouse $D l$ cause ARHED (autosomal recessive HED) and ADHED (autosomal dominant HED), which are clinically indistinguishable from the more common XLHED (Monreal et al., 1999).

With the recent cloning of the $D l$ gene (Headon and Overbeek, 1999), it is now possible to follow its expression during tooth development, and to compare it with that of its possible ligand, $\mathrm{Ta}$. We have shown that in the developing tooth $D l$ is expressed in the oral epithelium from E10, with a highly localised later expression in the epithelially derived enamel knot. This structure is thought to act as a signalling centre in the tooth, controlling proliferation and apoptosis, leading to the development of cusps (Jernvall et al., 1994; Vaahtokari et al., 1996a,b; reviewed in Tucker and Sharpe, 1999).

Recently, $T a$ expression was investigated in developing teeth, and was shown to be expressed from the cap stage (E14) in the oral epithelium and the outer enamel epithelium, but was absent from the inner enamel epithelium and mesenchyme (Srivastava et al., 1997; Pispa et al., 1999; http://honeybee.helsinki.fi/toothexp/TABBY.htm). $\quad \mathrm{Ta}$ is therefore not expressed in the enamel knot, but in the epithelium surrounding it. Expression of Ta prior to E14 has not been previously identified; however, we were able to detect weak $\mathrm{Ta}$ expression from E12 in the oral epithelium. By using adjacent sections, we were able to compare $T a$ and $D l$ expression domains, at these early stages, and show that they are largely mutually exclusive. Since both Eda and Edar are membrane bound, this suggests that the proteins are unlikely to interact directly in vivo, unless at least one of them is able to be cleaved to produce a secreted form.

In downless and tabby mutants, the teeth are severely affected, with very shallow depressions forming instead of the normal deep cusps in the molar region. We compared the early stages of tooth development in downless embryos with that recently reported for tabby (Pispa et al., 1999). We identified a failure in the formation of the enamel knot in downless embryos, where enamel knot cells adopt a sheet-like structure which we term the 'enamel rope'. Normal expression levels of enamel knot markers, such as Fgf4, Shh, Bmp4 and Wnt1Ob (Jernvall et al., 1994; Vaahtokari et al., 1996b; Dassule and McMahon, 1998), are retained, but the expression domains are disrupted. This phenotype is different to that observed in the tabby mutants, where a normal shaped enamel knot forms, but it is smaller and the expression of marker genes is weaker (Pispa et al., 1999). These different enamel knot phenotypes, together with the different expression patterns prompted us to determine whether Eda and Edar interact in vivo by using a soluble form of Edar added to tooth-germ explants to sequester endogenous Eda. The resulting effects on enamel knot formation suggests that Edar indeed interacts with Eda, although this interaction alone cannot fully explain the downless phenotype. The abnormal formation of the enamel knot signalling centre in downless and tabby mutants, linked to the resultant cusp defects in the adult, provides functional evidence that the enamel knot has a direct role in cusp morphogenesis.

\section{MATERIALS AND METHODS}

\section{Mutant mice}

Two mutants of spontaneous origin are known for downless, $d l^{\text {Jackson }}$ and $D l^{\text {Sleek }} . D l^{\text {Sleek} /+~ m i c e ~ h a v e ~ t h e ~ s a m e ~ p h e n o t y p e ~ a s ~} d l^{J} / d l^{J}$ animals. The $d l^{\mathrm{J}}$ mutation is caused by a single base pair change resulting in a glutamate to lysine substitution within the predicted death domain (Headon and Overbeek, 1999). $D l^{\text {Sleek }}$ mice produce a truncated transcript. The sleek mutation is thought to act as a dominant negative, binding to the ligand and wild-type receptor but lacking the cytoplasmic sequences required for signal transduction (Headon and Overbeek, 1999). dl Jackson mice were used in all the experiments described here.

\section{Preparation of embryonic tissue}

Mutant and wild-type heads from E10 to E18 were wax embedded and sectioned at $5 \mu \mathrm{m}$. Sections were split over 5-10 slides and prepared for radioactive in situs, TUNEL staining or Eosin/ Haemotoxylin staining.

\section{Preparation of adult tissue}

Eight-week-old adult jaws were dissected out and boiled to remove any soft tissue. Jaws were then photographed and the teeth removed to allow assessment of root and crown development.

\section{In situ hybridisation}

Radioactive ${ }^{35} \mathrm{~S}$ in situ hybridisation procedures were carried out as described by Wilkinson (1995), with modifications as described by Tucker et al. (1999). The antisense probes used were generated from mouse cDNA clones. $T a(03)$ was linearised with $X b a 1$ and transcribed with T7. $D l$ was linearised with BssHI and transcribed with T7. $p 21$ was linearised with BamHI and transcribed with T7. $F g f 4$ was linearised with Xmn1 and transcribed with SP6. Wnt $10 B$ was linearised with $X h o 1$ and transcribed with T3. Shh was linearised with EcoRI and transcribed with T7. Bmp4 was linearised with EcoRI and transcribed with SP6.

\section{TUNEL assay}

Apoptotic cells were localised by detecting DNA fragmentation. A digoxigenin-based modification of terminal deoxynucleotidyl transferase mediated labelling (TUNEL) was used for histological sections, using protocols described in Vaahtokari et al. (1996a). Slides were counter stained with Malachite Green or Eosin.

\section{Recombinant proteins}

The Edar soluble receptor was constructed by fusing the extracellular domain of murine Edar (amino acids 1-183) to the hinge and Fc portion of human IgG1. Human Fas (amino acids 1-170), TRAIL-R2 (amino acids 1-212) and 4-1BB (amino acids 1-186) were also expressed as Ig fusion proteins. cDNA for the TNF homology domain of murine Eda was amplified by nested PCR from mouse lung cDNA using primer pairs JT995 5' - GGATTCCAGGAACAACTGTTATGG3' JT996 5'-CCTACACACAGCAAGCACCTTAGAG-3' and JT997 5'-GTCGACGAAAATCAGCCAGCTG-3' JT998 5'-AAGCTTCTAGGATGCAGGGGC-3'. The TNF homology domain of murine Eda (amino acids 245-391) was cloned $3^{\prime}$ of the haemaglutinin signal peptide and of a Flag tag. Flag-tagged FasL (amino acids 103-281), TRAIL (amino acids 95-281) and 4-1BBL (amino acids 85-253) were also used. Construction, expression and purification were carried out as described by Schneider (2000).

\section{In vitro test}

The various receptors: $\mathrm{Fc}$ fusion proteins $(0.5 \mu \mathrm{g}$ each), were mixed with the different Flag ligands (about $200 \mathrm{ng}$ ) in $1 \mathrm{ml}$ of PBS and immunoprecipitated with $5 \mu \mathrm{l}$ of Protein A-Sepharose for 1 hour at $4^{\circ} \mathrm{C}$. Beads were loaded on mini-columns, washed twice with $400 \mu \mathrm{l}$ of PBS and eluted with $15 \mu \mathrm{l}$ of $0.1 \mathrm{M}$ citrate- $\mathrm{NaOH} \mathrm{pH}$ 2.7. Eluates 
were neutralised with Tris- $\mathrm{HCl}(\mathrm{pH} 8.5)$, resolved on $12 \%$ acrylamide gels and the co-precipitating ligands were detected by western blotting using anti-Flag M2mAb (1 $\mu \mathrm{g} / \mu \mathrm{l})$, horse radish peroxidase-coupled anti-mouse antibodies and ECL (Amersham-Pharmacia). The same blot was subsequently reprobed with horse radish peroxidase-coupled anti-human antibodies to reveal receptors:Fc.

\section{Treatment of tooth germ cultures with Edar soluble receptor}

Purified Edar:Fc was dissolved in D-MEM with glutamax (Gibco $\mathrm{BRL})+3 \% \mathrm{FCS}$ and $50 \mu \mathrm{g} / \mathrm{ml}$ apotransferin to give a final concentration of $50 \mathrm{ng} / \mathrm{ml}$ soluble receptor. Mandibular molar regions were dissected out from embryonic day (E) 12.5 embryos of the CD1 strain. Noon on the day on which the plugs were detected was considered as E0.5. Molars explants were cultured on membrane filters on metal grids following the Trowel technique as modified by Saxén (Trowel, 1959; Saxén, 1966) in a standard incubator at $37^{\circ} \mathrm{C}$ with an atmosphere of $5 \% \mathrm{CO}_{2}$ in air and $100 \%$ humidity. All solutions used contained penicillin and streptomycin at $20 \mathrm{IU} / \mathrm{ml}$. After three days the explants were washed in ice cold methanol for 1 minute then fixed in fresh $4 \%$ paraformaldehyde for 1 hour at room temperature. Cultures were then washed, taken through an ethanol series and embedded for sectioning.

\section{RESULTS}

\section{Expression of $\mathrm{DI}$ and $\mathrm{Ta}$ in tooth development}

Expression of $D l$ during early tooth development was followed by in situ hybridisation. $D l$ was first seen expressed in the oral epithelium at E10, which is a stage prior to thickening of the dental epithelium (Fig. 1A). Expression was strongest in the maxillary arch epithelium, with the mandibular arch expression not appearing until E10.5. At the same stage $D l$ was also strongly expressed in the apical ectodermal ridge (AER) of the limb (Fig. 1B). $D l$ expression became more restricted at E11 to the thickening dental epithelium, which was clearly seen by E12, when invagination of the epithelium starts to occur at the sites of tooth development (Fig. 1C,E). The earliest expression of $\mathrm{Ta}$ had previously been reported as E14, much later than we observed for $D l$ (Pispa et al., 1999). In order to confirm this we mapped $T a$ expression and found weak expression detectable in tooth epithelium at E12 (Fig. 1D,F). The expression of $\mathrm{Ta}$ was clearest in the incisor region on the medial (distal) side of the epithelial thickening (Fig. 1D). In contrast, $D l$ was expressed more laterally (proximally) within the thickening (Fig. 1C). The situation appears similar in molars, although the expression of $T a$ was weaker (Fig. 1E,F). This is the earliest expression so far shown for $T a$, although it is possible that $\mathrm{Ta}$ is expressed earlier, as suggested by northern blot analysis (Ferguson et al., 1997: Srivastava et al., 1997), but at such low levels that it can not be detected by in situ hybridisation.

At E13, the bud stage, $D l$ expression became restricted to the epithelial cells at the tip of the bud, the region thought to encompass the enamel knot precursor cells (Fig. 1G). The enamel knot is a transient structure consisting of a cluster of non-dividing cells at the site of the future first cusp. It has been associated with cuspal initiation in both incisors and molars (Butler, 1956). To see if $D l$ was indeed marking these cells its expression was compared to that of p21 ( Cdknla - Mouse Genome Informatics), a cyclin-dependent kinase inhibitor thought to be the earliest marker of the future enamel knot
(Jernvall et al., 1998). In reciprocal sections it was observed that the expression of $D l$ and $p 21$ in molar tooth germs corresponded exactly (Fig. 1H).

At E14, the cap stage, $D l$ was strongly expressed in the enamel knot of both the incisor and molar and appeared to be excluded from other regions of epithelium (Fig. 2A,C,E,G). Expression was also clear in the whisker follicles (Fig. 2A). At this stage $\mathrm{Ta}$ was expressed at the collar of the developing cap in the outer enamel epithelium and a restricted region of the oral epithelium (Fig. 2B,D,F,H). The expression patterns of $T a$ and $D l$ are almost complementary at this stage in the epithelium. This again is more obvious in the incisor region, with $T a$ being expressed in the epithelial cells nearest to the
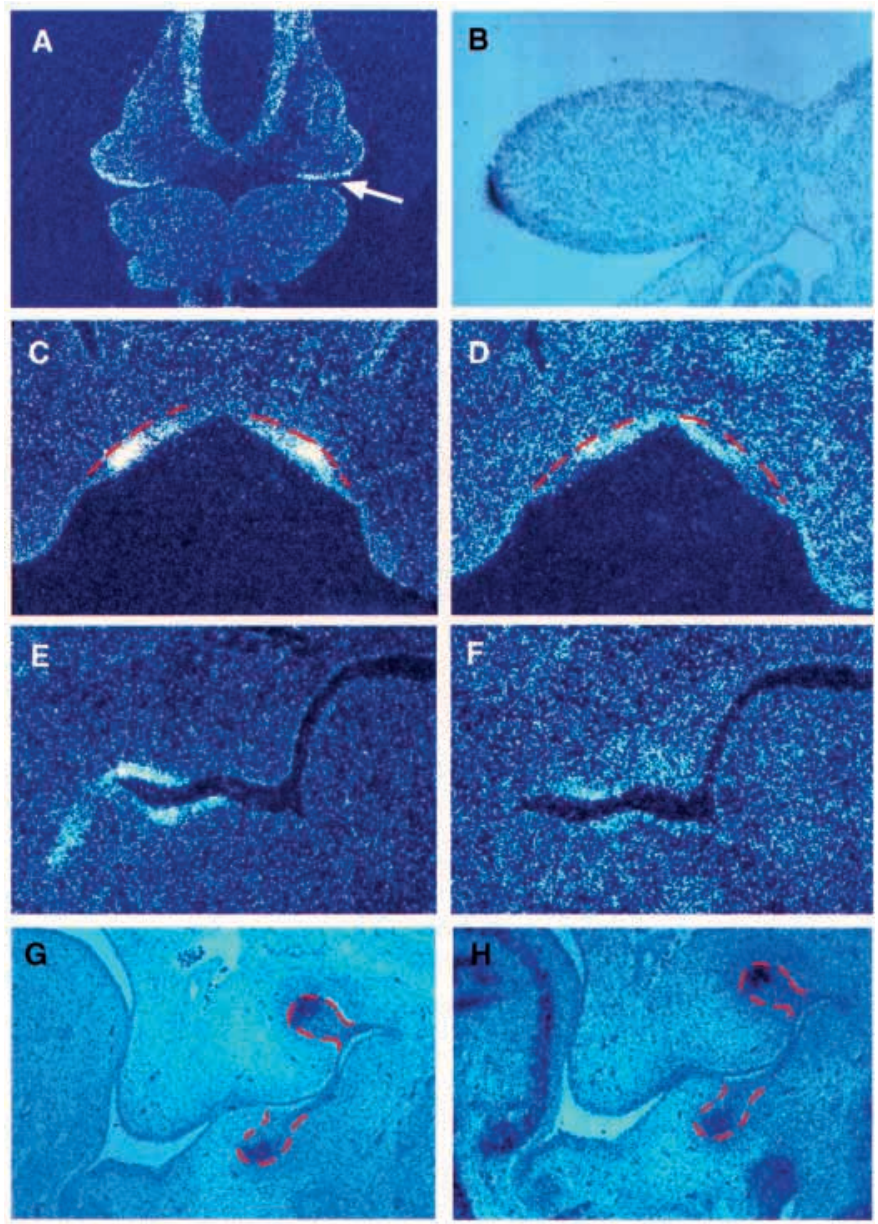

Fig. 1. Expression of $D l$ and $T a$ in early tooth development. ${ }^{35} \mathrm{~S}$ in situ hybridisation of $D l$ (A-C,E,G), $T a(\mathrm{D}, \mathrm{F})$ and $p 21(\mathrm{H})$.

(A) E10 frontal section through a mouse head, showing $D l$ expression in the oral epithelium (arrow). (B) E10 section through the forelimb, showing $D l$ expression in the apical ectodermal ridge (AER). (C) E12 frontal section showing $D l$ in the thickened epithelium in the maxilla incisor region. (D) Serial section showing $T a$ expression in the oral epithelium on the medial (distal) side of the thickening. (E) E12 frontal section through the molar region, showing high $D l$ expression in the thickenings. (F) Serial section showing Ta expression weakly in this region. (G) E13 frontal section through the molar region showing $d l$ expression at the tips of the invaginating epithelial buds. (H) Serial section showing p21 expression in an overlapping group of cells. Broken red lines outline the dental epithelium. 

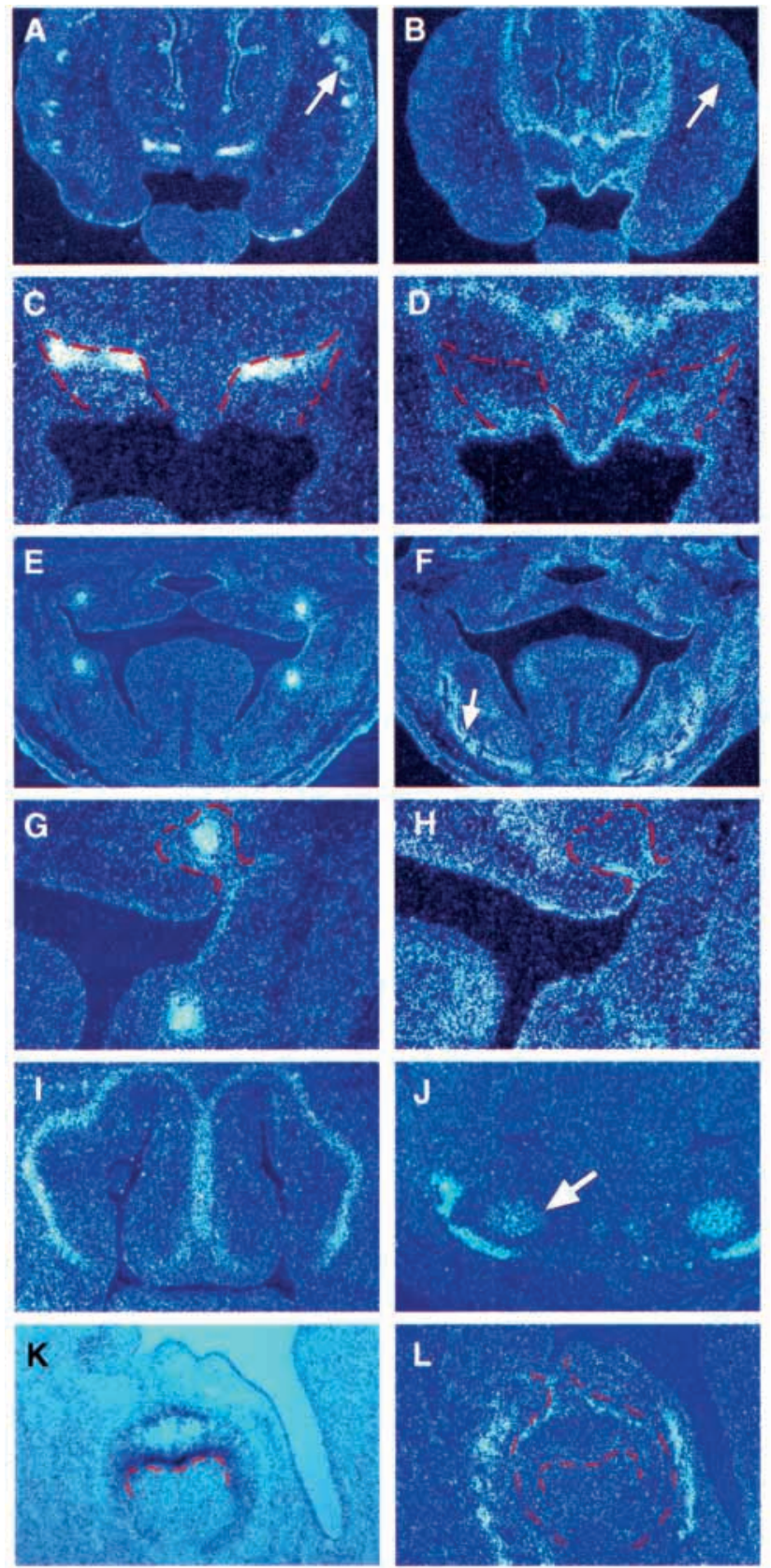

oral cavity and $D l$ being expressed in the epithelial cells nearest to the dental mesenchyme (Fig. 2C,D). Ta was also seen expressed in the forming glands of the face, such as the serous nasal glands and in the developing bone and cartilage (Fig. 2I,J). Interestingly, these glands are absent or reduced and the skull and facial bones dysmorphic in the tabby mutant (Grüneberg, 1971) and in patients with XLHED (Kere et al., 1996; Montonen et al., 1998). The distinct expression patterns of $T a$ and $D l$ were also observed at E18, with $D l$ being expressed in the inner enamel epithelial cells, and $\mathrm{Ta}$ being expressed in the outer enamel epithelium (Fig. 2K,L).

\section{Expression of $D I$ and Ta in tabby and Lef1 mutant mice}

In tabby mutant mice at E13.5, $D l$ expression was found to be normally distributed, indicating that the induction of $D l$ is not
Fig. 2. Expression of $D l$ and $T a$ at the cap and bell stage. ${ }^{35} \mathrm{~S}$ in situ hybridisation of $D l$ (A,C,E,G,K) and $T a$ (B,D,F,H,I,J,L). (A,B) E14 frontal section through distal region of head showing cap stage incisors. (A) $D l$ is expressed in the incisors and whisker follicles (arrow). (B) $T a$ is expressed in the incisors and linking oral epithelium. Weak expression is observed uniformly around the whisker follicles (arrow). (C,D) Close up of incisor cap stage tooth germs, showing $\mathrm{Dl}$ at the border between the inner enamel epithelium and the dental mesenchyme, and $T a$ at the base of this region in the epithelial cells closest to the oral cavity. (E,F) E14 frontal section through proximal region of head showing cap stage molars. (E) $D l$ is expressed in the enamel knots, at the centre of the tooth germ. (F) $\mathrm{Ta}$ is expressed in a collar at the base of the tooth germ in the outer enamel epithelium. Expression is also seen in developing bone (arrow). (G,H) Close up of molar cap stage tooth germs. (I,J) Close up of $\mathrm{Ta}$ expression in the nasal glands (I) and developing bone and cartilage (J). Arrow indicates the developing rod of Meckel's cartilage. (K,L) E18 frontal section through bell stage molar tooth germs. (K) Expression of $D l$ in the inner enamel epithelium adjacent to the dental mesenchyme.

Expression is also seen near to the outer enamel epithelium at the base of the tooth germ. (L) Expression of $T a$ in the outer enamel epithelium at the base of the tooth germ, and in the surrounding bone. Broken red lines outline invaginating epithelium.

reliant on the presence of active Ta protein (Fig. 3A, compared to $1 \mathrm{G})$. At E13.5 the Ta phenotype, of a smaller tooth germ is only just evident (Pispa et al 1999). This agrees with the fact that $D l$ appears to be expressed prior to $T a$ in the oral epithelium.

It has previously been suggested that the tabby pathway might involve the transcription factor Lef1. Lef1 is a member of the HMG protein family which has the capacity to induce sharp bends in the DNA helix (Giese et al., 1992). Lefl mutants have severe defects in teeth, mammary gland and whisker development, together with abnormal development of many hair follicles (van Genderen et al., 1994). From recombination experiments it has been shown that it is the epithelial expression of Lefl that is responsible for these defects (Kratochwil et al., 1996). The Ta gene has a potential Lef1binding site in its promoter, indicating that $T a$ may lie downstream of Lef1 (Zou et al., 1995; Kere et al., 1996).

To investigate the interaction between Lefl and $T a$ and $D l$, the expression of these genes were compared in wild-type and Lefl mutant mice at E13 (the stage at which tooth development is arrested in Lefl mutants). $D l$ and $T a$, expression were found to be normal in Lefl mutant tooth buds, suggesting that in tooth development Lef1 is not upstream of the Ta/Edar pathway (Fig. 3B-D, compared with Fig. 1G).

\section{Phenotypic defects during tooth development in downless mutants}

In downless mice, the incisors and molars have been noted as appearing abnormal or missing (Sofaer, 1969a,b; 1977). To investigate this phenotype further we examined adult teeth in $d l^{\text {Jackson }}$ homozygous and $\mathrm{Dl}^{\text {Sleek }}$ heterozygous mice 8 weeks after birth. In the mutant examples investigated all incisor and molar teeth were present. The incisors were of normal shape and size but the molars, particularly the first, were much reduced in size compared with wild-types and had a flattened appearance (Fig. 4A,C). Thus, instead of the deep cusps seen in the wild-type mice, very shallow depressions were observed. In the downless mutant only three of the normal eight cusps 

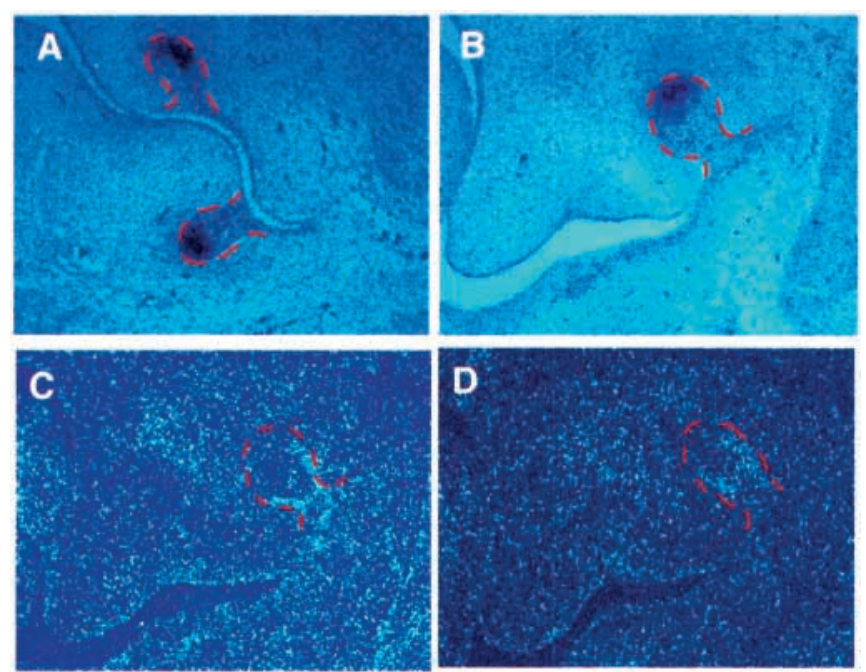

Fig. 3. Expression of $D l$ and $T a$ in tabby mice and Lefl mutant mice. ${ }^{35} \mathrm{~S}$ in situ hybridisation of $D l(\mathrm{~A}, \mathrm{~B})$ and $T a(\mathrm{C}, \mathrm{D})$. (A) E13 frontal section through molar region at the bud stage, showing normal expression of $D l$ in tabby mutant (compare with Fig.1G). (B) E13 frontal section through molar region at the bud stage showing normal expression of $D l$ in Lefl knockout (compare with Fig.1G). (C) Wildtype section showing expression of $T a$ at the bud. (D) Serial section showing normal expression of Ta in Lefl knockout. Broken red lines outline invaginating epithelium.

formed in the first upper molars and four of the normal seven cusps in the lower first molars. A more severe phenotype in molars compared to incisors has also been noted in tabby mice (Grüneberg, 1965). A tabby incisor phenotype, however, was identified after careful 3D reconstruction of the lower incisors (Miard et al., 1999). No obvious differences were evident between $d l^{\text {Jackson }}$ and $D l^{\text {sleek }}$ adult molar teeth (Fig. 4D) although variations in the severity of the cusp defects have been reported (Sofaer, 1969b). The defects observed were identical to those previously described for $D l^{\text {sleek }}$ (Sofaer, 1977) and for tabby (Grüneberg, 1965, 1966), and were similar to those seen in XLHED in humans (Clarke, 1988 - DM thesis, Oxford University; Crawford et al., 1991).

During embryogenesis, the development of the $d l^{\text {Jackson }}$ homozygous mutant teeth appeared relatively normal up to the cap stage E14-E15. At this point, the enamel knot, visible as a thickened bulge of cells at the heart of the tooth in wild-type molars, was not apparent (Fig. 4E,F). The overall size of the tooth germs, however, appeared normal at this point.

\section{Conversion of the enamel knot into an 'enamel rope'}

To investigate the enamel knot defect in more detail, the expression of genes associated with this signalling centre were compared between wild-type and $d l^{\text {Jackson }}$ homozygous mutant mice. At E13, when the enamel knot is starting to form, the expression pattern of $p 21$ and $D l$ were found to be disrupted. Normally these genes are expressed at the tips of the buds in the molar region (Fig. 1G,H). In the downless mutant, expression was diffuse and spread throughout the invaginating epithelium. This effect was most obvious in the maxillary molars. The expression of $p 21$ also appeared downregulated in the buds when compared with the normal levels seen in other structures such as the tongue. The surrounding condensing
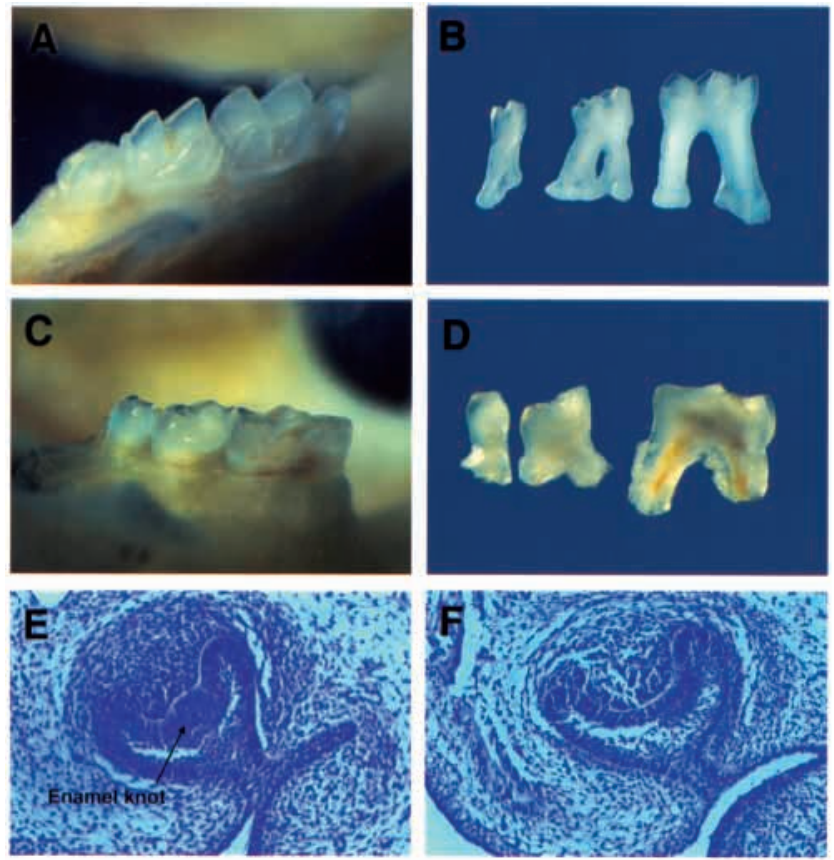

Fig. 4. Tooth phenotype of $D l$ mutant. (A,B) Wild-type mandibular teeth at 8 weeks. (C,D) $D l$ mutant mandibular teeth at 8 weeks. (A) Teeth embedded in jaw showing deep and defined cusps. (B) Teeth showing multiple, substantial roots. (C,D) Teeth with reduced and rounded cusps and reduced roots. (E,F) E15 molar tooth germs at cap stage. (E) Wild-type molar tooth germ with obvious enamel knot clustering of cells at the centre of the invaginated epithelium. (F) Loss of physical enamel knot in $d l$ mutant.

mesenchymal cells, however, appeared relatively normal as shown by the expression of Bmp4 (Fig. 5C,D), a gene thought to be responsible for induction of the early enamel knot at the bud stage (Jernvall et al., 1998). The expression was slightly more diffuse, especially in the upper molar but by E14.5 however, expression appeared normal in the mesenchyme (Fig. $6 \mathrm{~J})$.

At E15, genes known to be expressed in the enamel knot were compared. It was assumed that expression of such genes would be absent or downregulated, reflecting the loss or reduction of the enamel knot, but instead expression was at wild-type levels but stretched as an elongated band of cells $(n=8)$. This was clearly seen for Fgf4, which is usually expressed only in at the centre of the enamel knot (Jernvall et al., 1994; Fig. 6A,B), and for Shh, which is usually expressed over a slightly larger area, correlating with the non-dividing cells (Vaahtokari et al., 1996b; Fig. 6C,D). Expression of Wnt10b, which overlaps with that of Shh in the enamel knot, was also similarly affected and could be seen to extend in an elongated epithelial structure very different from the enamel knot (Dassule and McMahon, 1998; Sarkar and Sharpe, 1999; Fig. $6 \mathrm{E}, \mathrm{F}) . \mathrm{Dl}$ itself was also shown to follow this pattern of expression (Fig. 6G,H). These changes were seen in all $\mathrm{dl}^{\text {Jackson }}$ embryos examined with some variation in the extent of the enamel rope. Such variation may relate to the variation in the severity of cusp defects reported. Thus, enamel knot cells are formed but their organisation is dramatically affected by the absence of downless protein. The expression of Bmp4 in the condensing mesenchyme seemed to be normal in the mutant 

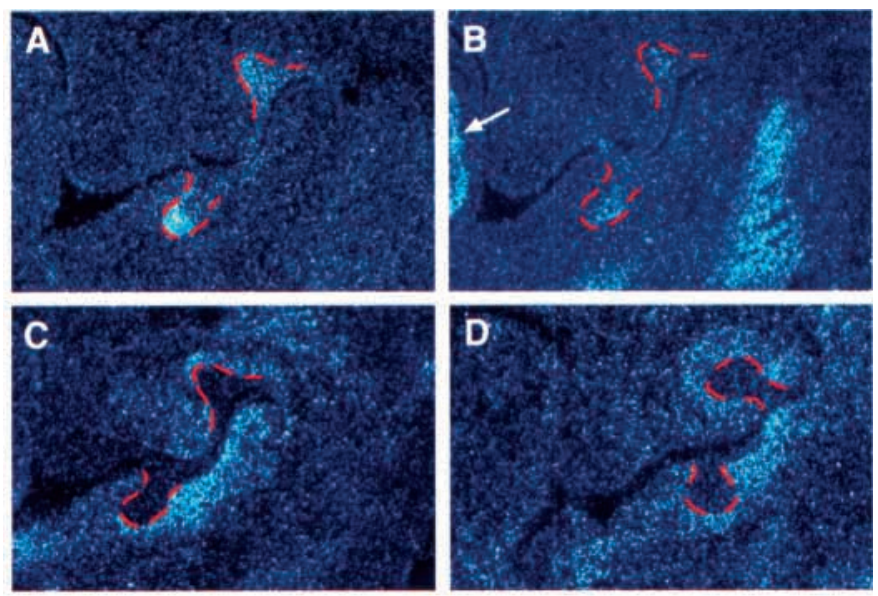

Fig. 5. Irregular formation of the early enamel knot in $\mathrm{Dl}$ mutant. ${ }^{35} \mathrm{~S}$ radioactive in situ hybridisation of the molar tooth bud at E13. (A) Diffuse expression of $D l$ at the tip of the invaginating epithelium in $D l$ mutant (compare expression with Fig. $1 \mathrm{G}$ ). Note the defect in the upper is more pronounced than the lower molar, with some accumulation of signal at the tip of the bud in the lower tooth bud. (B) Serial section showing diffuse and weak expression of $p 21$ in an overlapping domain in $\mathrm{Dl}$ mutant (compare expression with Fig. $1 \mathrm{H}$ ). Note again the defect is more pronounced in the upper molar. Expression in the tongue appears normal (see arrow). (C) Serial section showing normal Bmp4 expression in the mesenchyme surrounding the invaginating epithelium in the $D l$ mutant.

(D) Expression of Bmp4 in a wild-type embryo. Broken red lines outline invaginating epithelium.

tooth germs, but the expression in the epithelial cells overlying this region was similar to $S h h, F g f 4$ and Wnt $10 b$ (Fig. 6I,J). Ta expression in the collar region of the tooth was also found to be normal (data not shown), indicating that $\mathrm{Ta}$ does not need functional downless protein to maintain its expression pattern. The levels of expression of the enamel knot markers appeared normal, compared with controls. This is in contrast to the tabby mutant mice, where expression is found at a much lower level (Pispa et al., 1999).

At E18 the secondary enamel knots form in the molars. These secondary enamel knots form above where additional cusps are to develop. Incisors do not develop secondary knots, as predicted by their monocuspid morphology. The secondary knots are highlighted by the expression of Fgf4, which moves from the centre of the tooth to the site of two new cusps (Jernvall et al., 1994). The presence of secondary enamel knots was compared between wild-type and downless mutant mice. Instead of two compact groups of cells expressing $\mathrm{Fgf4}$ at the position of the new knots, Fgf4 was now expressed in a band between the normal two sites (Fig. 6K,L). Thus, no normal secondary enamel knots were formed, helping to explain the reduced number of cusps seen in downless adult teeth. Again Ta expression was found to be normal (Fig. 6M,N). Overall, the tooth germs at E18 appeared smaller than the wild-type.

\section{Eda and Edar interact in vitro}

The difference in expression pattern and enamel knot defect between tabby and downless mutant mice, raised the question of whether Eda and Edar do really interact directly. Using recombinant, soluble forms of Eda and Edar, a strong and specific interaction was readily demonstrated, which was comparable with those of three other pairs of ligands and receptors of the TNF family, namely Fas/FasL, TRAILR2/TRAIL and 4-1BB/4-1BBL (Fig. 7). Recombinant Eda migrated as a double band, which is most probably the result of differential $\mathrm{N}$-glycosylation. It did not interact with 22 other receptors of the TNFR family, and Edar:Fc failed to immunoprecipicate 12 further ligands of the TNF family (data not shown).

\section{Soluble forms of Edar phenocopy the tabby tooth phenotype}

We tested the effect of adding soluble forms of Edar to explant cultures of mandibular molars at E12.5. At this stage the tooth germ is visible as a deep thickening (see Fig. 1C), and no defects are yet obvious in the downless or tabby mutant mice. The cultures were left for three days to develop to the cap stage, when the enamel knot becomes prominent. Soluble forms of other TNF receptors have been shown to be able to inhibit the activity of TNF ligands in cell culture (Chen et al., 1997), therefore, we predicted that addition of the soluble Edar would inhibit endogenous Eda. The resulting tooth germs had small enamel knots, which expressed sonic hedgehog $(S h h)$ at a greatly diminished extent (Fig.8A,B,D,E). The expression of Fgf4 appeared to be completely lost when compared with control cultures (Fig. 8C,F). The reduction of Shh expression was particularly noticeable along the longitudinal axis, as indicated by counting the number of sections showing gene expression. In controls the expression of Shh appeared to last for at least $60 \mu \mathrm{m}$, while expression in the Edar treated cultures appeared across only $30 \mu \mathrm{m}$. The loss of Fgf4 might indicate a delay in development of the treated cultures, when compared to controls, since Fgf4 is expressed in the enamel knot after Shh. The expression of other genes not associated with the enamel knot, for example $L h x 7$, appeared unaffected by the treatment indicating that the soluble receptor was not having a non-specific effect (Fig. 8D,G). This phenotype is strikingly similar to the tabby phenotype (Pispa et al., 1999). Therefore, addition of soluble Edar disrupts the Eda pathway, but fails to reproduce the downless phenotype.

\section{Apoptosis is not affected in downless mutant tooth germs}

The death domain, which is disrupted in downless Jackson mutants, was named due to its frequent involvement in transduction of apoptotic signals (Ashkenazi and Dixit, 1998). The enamel knot is a well-established localised site of apoptosis, which leads to its eventual silencing as a signalling centre (Vaahtokari et al., 1996a). Apoptosis starts at the bud stage, correlated with first expression of the enamel knot cell marker $p 21$ (Jernvall et al., 1998). The extent of apoptosis seen in bud stage tooth germs in wild-type and downless mutant mice was compared using a TUNEL assay (Fig. 9A,B). No change in the number of apoptotic cells was seen at this stage, indicating that the downless phenotype is not caused by a change in cell death within the tooth germs, as might be predicted by the reduced expression of $p 21$. No change in cell death agrees with the fact that loss of $D l$ does not result in an enlargement or loss of the enamel knot but rather a change in its shape. It is possible the death domain in $D l$ may be involved in mediating more general protein-protein interactions (Newton et al., 1998; Feinstein et al., 1995). 

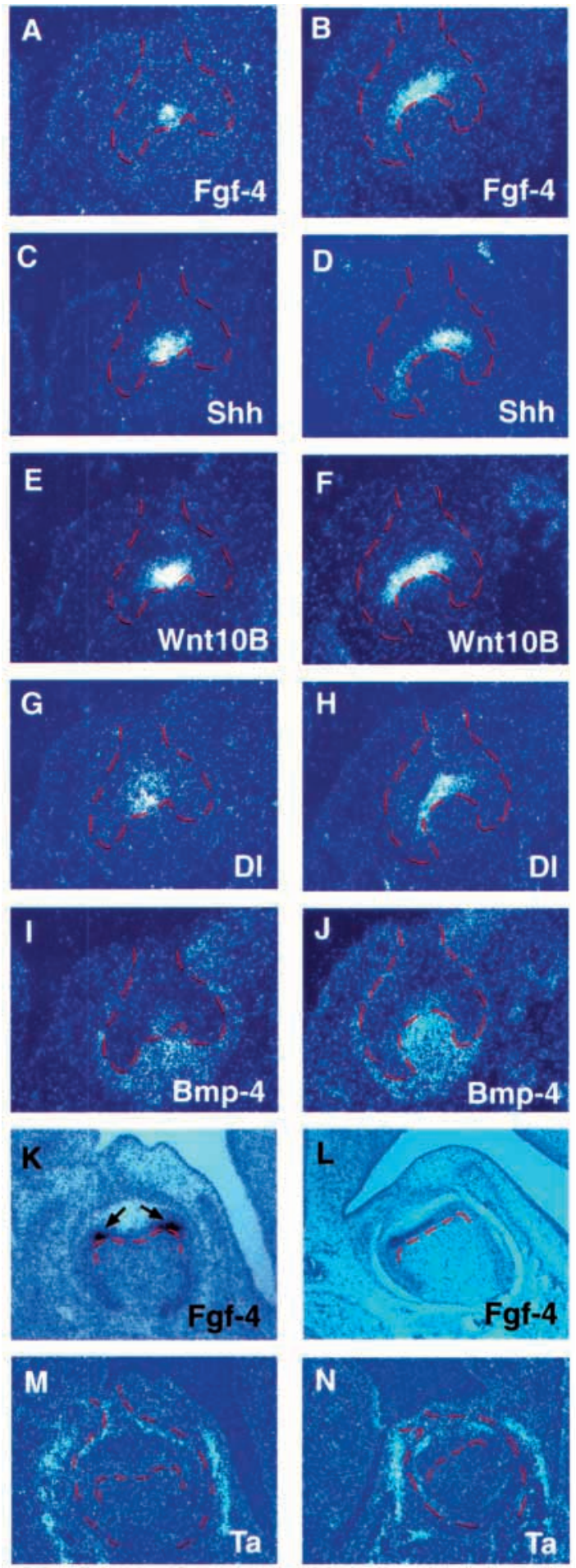

\section{DISCUSSION}

The similar adult phenotypes of tabby and downless mutants indicated that $T a$ and $D l$ work within the same pathway. This is supported by the fact that Eda is a member of the TNF family of membrane-bound ligands, while Edar belongs to the TNFR family of membrane-bound receptors. However, the expression patterns of the two genes show that $T a$ and $D l$ are not expressed in overlapping domains within the tooth epithelium, and in fact
Fig. 6. Conversion of the enamel knot to an enamel rope. ${ }^{35} \mathrm{~S}$ radioactive in situ hybridisation showing the molar region at the cap stage. (A-J) E15 Cap stage. (K-N) E18 Bell stage. (A,C,E,G,I,K,M) Wild-type serial sections. (B,D,F,H,J,L,N) Dl mutant serial sections. (A) Fgf4, (C) Shh, (E) Wnt1Ob, (G) Dl expression concentrated in the enamel knot. (B) Fgf4, (D) Shh, (F) Wnt 1Ob, (H) Dl expression in a band of cells along the inner enamel epithelium. (I) Bmp4 expression in the enamel knot and in the condensed dental mesenchyme.

(J) Bmp4 expression shows normal expression in the mesenchyme but the epithelial expression is spread out over the inner enamel epithelium, as for the other enamel knot markers. (K) Fgf4 expression in the secondary enamel knots (arrows).

(L) Fgf4 expression spread out between the two normal sites of expression. (M) $\mathrm{Ta}$ expression in the collar region of the tooth germ in the outer enamel epithelium, and in the surrounding bone in a wild-type embryo. (N) Normal Ta expression in a downless mutant. Broken red lines outline dental epithelium.

appear largely mutually exclusive (Figs 1 and 2). Eda has recently been found to contain a protease recognition site (furin) that may cleave to generate a secreted form of the protein (Ferguson et al., 1998). A similar furin cleavage site is found in BAFF, APRIL and Tweak, the three TNF ligands most related to Eda, and solubilisation of BAFF at this site has been demonstrated (Schneider et al., 1999). In this way, Eda may act as a diffusable ligand for Edar. However, when Eda was transfected into cells no evidence of shedding from the plasma membrane was seen (Mikkola et al., 1999). Further experiments are required to clarify this issue.

Although the adult phenotypes are identical, we show that the defects at the cap stage of tooth development are strikingly different. In tabby the enamel knot appears to have a normal shape but is reduced in size (Pispa et al., 1999). In the downless

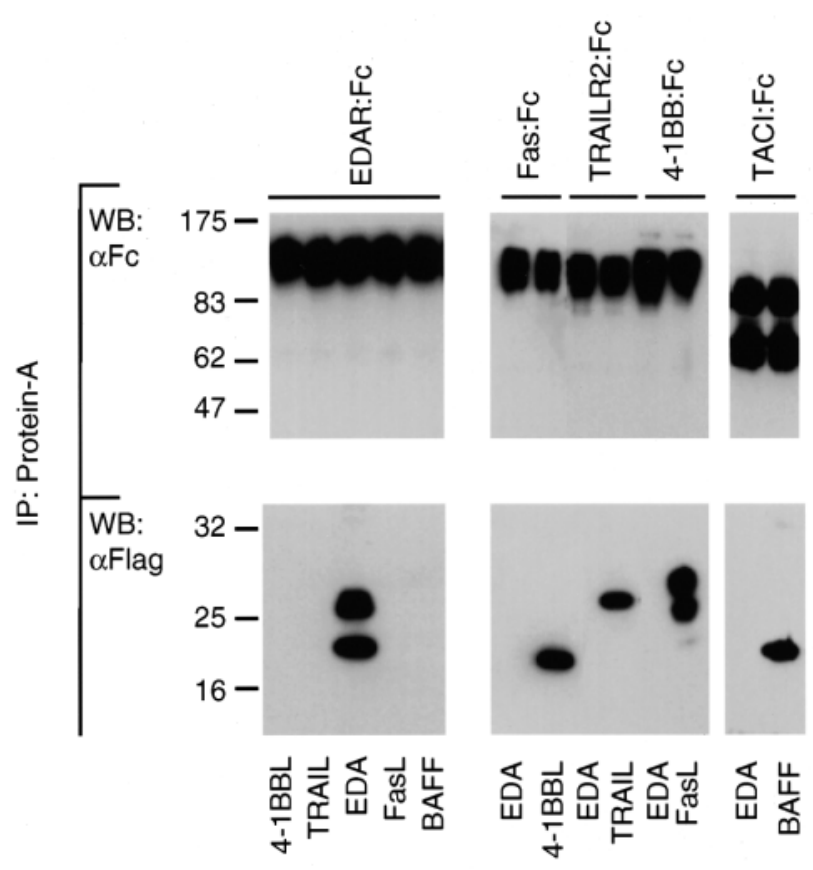

Fig. 7. The extracellular domain of Edar interacts with Eda. Murine Edar:Fc and control receptors:Fc fusion proteins were mixed with Flag-tagged Eda or control Flag-tagged ligands. Receptor:Fc are revealed with anti-human $\operatorname{IgG}$ (upper panels) and co-precipitating ligands are detected using anti-flag M2 antibody (lower panels). 
Fig. 8. Phenocopy of the tabby mutant using soluble Edar.

(A-D) Control E12.5 molar explants cultured for 3 days. (E-H) Explants cultured in the presence of soluble Edar. (A,E) Haemotoxylin/Eosin stain showing cap stage tooth germ. $(\mathrm{B}, \mathrm{F}) S h h$ expression. Note dramatic loss of Shh expression in the treated enamel knot. (C,G) Fgf4 expression. Note loss of expression of Fgf4 in the treated enamel knot. (D,H) $L h x 7$ expression. Note normal levels of expression in the treated

mesenchyme. Tooth germ is outlined in white.
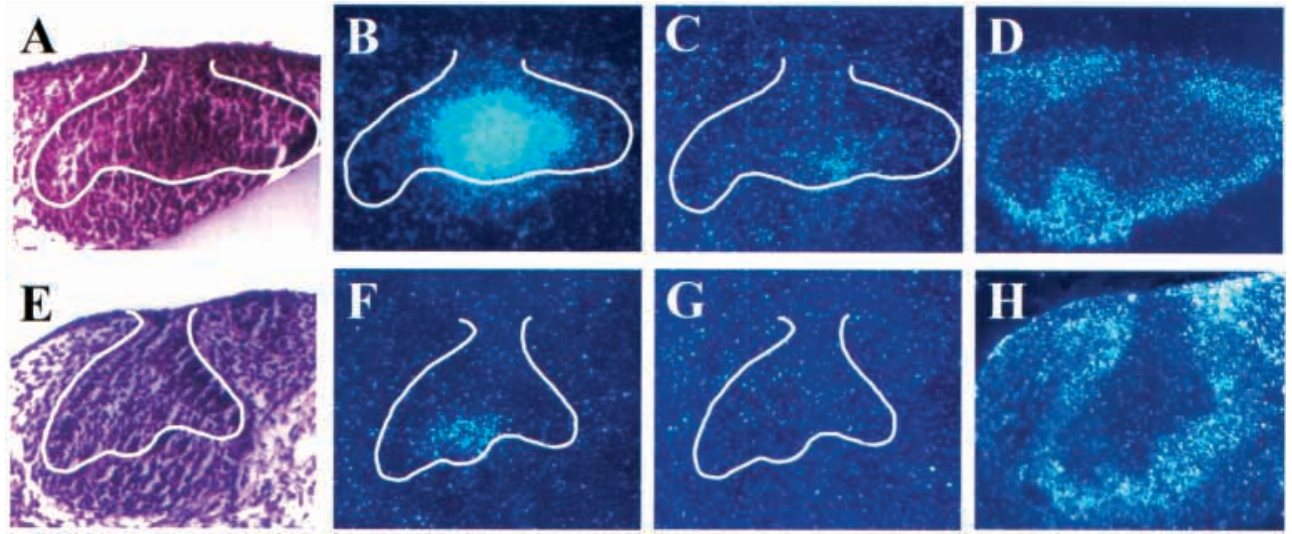

mutant the enamel knot is lost, and the enamel knot cells spread out along the inner enamel epithelium (Fig. 4 and 6). Tabby mice, therefore, have small enamel knots while downless mice have disorganised enamel knot cells. That these two different defects lead to almost identical phenotypes of small teeth with reduced cusps, indicates that the enamel knot is failing to function effectively in both cases. Even if solubilisation of Eda does occur, the different enamel knot defects suggest that the simple interaction where Eda binds and activates Edar, is not occurring.

To further investigate the ability of Eda and Edar to interact, fusion proteins were created and their ability to immunoprecitate together was assessed. In this in vitro study, Eda and Edar were found to bind specifically (Fig. 7). When tooth germs were treated with soluble Edar they developed small tabby-like enamel knots, rather than the enamel ropes of the downless mutant (Fig. 8). That soluble Edar can phenocopy tabby indicates that in the cultures it is blocking endogenous Eda.

The fact that the soluble Edar failed to reproduce the downless phenotype indicates the presence of additional components in the pathway. For example, Edar may be activated by more than one ligand, in which case removal of Eda is not sufficient to arrest Edar signalling. This second ligand might be membrane bound, possibly on the same cell as the receptor, and therefore would be unaffected by the soluble Edar. In this situation, the binding of ligand to receptor would act to hold enamel knot cells together, a mechanism consistent with the spreading of enamel knot cells in downless cap stage tooth germs. Alternatively, the penetration of the soluble receptor into the culture may have been limited, so that the endogenous ligand for Edar was protected at the enamel knot deep within the tooth germ, while the more superficially expressed Eda was more accessible. One possible candidate for an additional component in the Eda/Edar pathway might be the product of the crinkled gene. The teeth of crinkled mutants have similar defects to those seen in tabby and downless (Grünberg, 1965, 1966), and epidermal-dermal recombinations have identified the epidermis once again as the site of action of the gene (Mayer et al., 1977). crinkled has been mapped to Chromosome 13, thus it is independent of the downless (chromosome 10) and tabby (X chromosome) locus. It would seem likely that crinkled will also affect the formation of the enamel knot during tooth development, and it will be interesting to test this possibility once the gene has been characterised.
Rather than indicating the presence of a second ligand, Edar may have ligand-independent activity, as has been indicated to occur in other members of the TNFR family (Micheau et al., 1999). In this case, removal of Eda by a soluble receptor, would not be expected to produce the same phenotype as a block of Edar function, as is predicted to be occurring in the downless mutant. Finally, we cannot exclude the possibility that the difference in phenotypes between tabby and downless might simply indicate that the two genes do not work within the same pathway in vivo, and that the ability of soluble Edar to bind Eda is an artefact.

$D l$ is an early marker for the developing enamel knot during tooth development (Figs 1 and 2). In the downless mutant a defect is first seen at the bud stage when the enamel knot is first forming, as shown by altered expression of early markers such as $p 21$ (Fig. 5). By the cap stage, when the wild type knot is visible as a clustering of cells, the mutant cells fail to adopt their usual distribution and instead form an elongated sheet of cells that we have termed the 'enamel rope'. This structure expresses many of the same genes as the enamel knot, such as Shh, Bmp4, Fgf4, Wnt $10 b$ and Dl itself, at the same high levels, but in a different spatial pattern (Fig. 6). Expression of these genes can therefore not be linked directly with Edar function. Interestingly, it has recently been shown that in downless mutant epidermis the expression of Shh and Bmp4 are lost (Headon and Overbeek, 1999). Such differences between the tooth and the hair follicles are also seen in other knockouts. For example, loss of epidermal growth factor receptor function affects only the hair follicles,
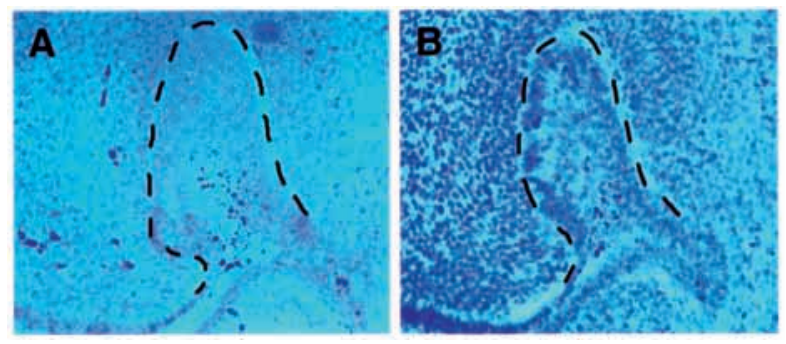

Fig. 9. Normal apoptosis at the bud stage in downless mutants. TUNEL assay showing apoptotic cells as blue grains. (A) Wild-type apoptosis concentrated at the centre of the invaginating tooth germ. (B) Comparable apoptosis in downless mutant. Broken lines outline invaginating epithelium. 
despite being expressed in both the developing follicles and the tooth germs.

The disruption of the enamel knot into an enamel rope correlates with cusp defects in the developing teeth, directly linking the need for the enamel knot with cusp morphogenesis (Fig. 4). The limited defect observed in incisor teeth, compared with molars, indicates that the enamel knot formed in incisor teeth has a slightly different role to that in the multicuspid molars. In normal development, the cusps that start to from first become the highest, with subsequently forming cusps being progressively smaller (Jernvall, 1995). The areas that are abnormal in the lower molars of the downless mutant are generally those that develop late, such as L1 (lingual cusp 1) (Sofaer, 1969b). Such later developing cusps may be more susceptible to a general reduction in size and complexity of the whole crown. The upper molars, however, do not fit in with this pattern, with loss of B3 (buccal cusp 3) occurring frequently, despite the early development of this cusp (Grüneberg, 1965). The fact that cusps do form in downless mutants suggests that the signalling functions of enamel knot cells are partially retained as shown by the expression of $F g f 4$, Shh and Wnt10b. The abnormal shape of the structure presumably slightly alters the action of these signalling factors leading to abnormal cusp formation.

The mechanism by which $D l$ is working to create an enamel knot is unclear although effects on apoptosis can be excluded. Eda increases the adhesion of transfected epithelial cells to extracellular matrix (Mikkola et al., 1998, 1999). A cell rounding effect has also been reported (Ezer et al., 1997), but this study used a form of Eda that did not contain the TNF domain or collagen domain, and as such this isoform is possibly nonfunctional. A defect in cell adhesion is also likely to be the cause of the downless enamel knot defect. Absence of functional $D l$ may result in failure of cells destined to form the enamel knot to adhere together, resulting in a flattened sheet-like structure instead. The possibility that the Edar ligand is expressed on the same cells as Edar is consistent with a cellcell adhesion mechanism. Interestingly, EGF, which is able to partially rescue the tabby mutant phenotype, is also able to induce cell rounding in epithelial cells by counteracting the adhesive function of E-cadherin (Hoschuetzky et al., 1994). Thus EGF, working with Lef1, may induce rounding of cells in the enamel knot, in the absence of Ta. This points to the $D l / T$ a pathway(s) running independently of the Egf/Lef1 pathway. Such a parallel pathway has also been suggested to occur in hair follicles (Headon and Overbeek, 1999). We support these studies by showing that $D l$ and Ta expression is normal in Lefl knockout mice, despite the potential Leflbinding site in the Ta promoter (Fig. 3).

Loss of functional downless primarily leads to a failure of the enamel knot cells to retain their knot-like structure. This is likely to lead to secondary defects due to reduced efficiency of the compromised signalling centre. Since one of the functions of the enamel knot is to stimulate proliferation (Jernvall et al., 1994) it is therefore not surprising that there is an overall reduction in the size of the tooth germ in downless and tabby mutant teeth. In fact, it has been shown possible to rescue much of the tabby mutant cusp phenotype by addition of FGF4 on beads (Pispa et al., 1999). Such a rescue is thought to be due to the ability of FGF4 to stimulate epithelial proliferation (Jernvall et al., 1994).
We thank Bert Vogelstein for the p21 probe, Ivor Mason for the Fgf4 probe, Andy McMahon for the Shh probe, Brigid Hogan for the Bmp4 probe, Phil Leder for the Wnt10b probe and Rudi Groschehl for the Lefl knockout mice.

\section{REFERENCES}

Ashkenazi, A. and Dixit, V. M. (1998). Death receptors: signaling and modulation. Science 281, 1305-1308.

Bayes, M., Hartung, A. J., Ezer, S., Pispa, J., Thesleff, I., Srivastava, A. K. and Kere, J. (1998). The anhidrotic ectodermal dsyplasia gene (EDA) undergoes alternative splicing and encodes ectodysplasin-A with deletion mutations in collagenous repeats. Hum. Mol. Genet. 7, 1661-1669.

Butler, P. M. (1956). The ontogeny of molar pattern. Biol. Rev. 31, 30-70.

Chen, T. C., Hinton, D. R., Sippy, B. D. and Hofman, F. M. (1997). Soluble TNF-alpha receptors are constitutively shed and downregulate adhesion molecule expression in malignant gliomas. J. Neuropathol. Exp. Neurol. 56, 541-550.

Crawford, P. J. M., Aldred, M. J. and Clarke, A. (1991). Clinical and radiographic dental findings in X-linked hypohidrotic ectodermal dysplasia. J. Med. Genet. 28, 181-185.

Darwin, C. (1875). The variations of animals and plants under domestication. London: John Murray.

Dassule, H. R. and McMahon, A. P. (1998). Analysis of epithelialmesenchmal interactions in the initial morphogenesis of the mammalian tooth. Dev. Biol. 202, 215-227.

Ezer, S., Sclessinger, D., Srivastava, A. K. and Kere, J. (1997). Anhidotic ectodermal dysplasia (EDA) protein expressed in MCF-7 cells associates with cell membrane and induces rounding. Hum. Mol. Genet. 6, 1581-1587.

Falconer, D. S. (1952). A totally sex-linked gene in the house mouse. Nature 169, 664.

Feinstein, E., Kimchi, A., Wallach, D., Boldin, M. and Varfolomeev, E. (1995). The death domain: a module shared by proteins with diverse cellular functions. Trends Biochem. Sci. 20, 342-344.

Ferguson, B. M., Brockdorff, N., Formstone, E., Ngyuen, T., Kronmiller, J. E. and Zonana, J. (1997). Cloning of Tabby, the murine homologue of the human EDA gene: evidence for a membrane associated protein with a short collagenous domain. Hum. Mol. Genet. 6, 1589-1594.

Ferguson, B. M., Monreal, A., Headon, D., Overbeek, P. and Zonana, J. (1998). An apparent new member of the TNF superfamily is encoded for by the EDA1 gene: evidence from mutation and two-hybrid analysis. Am. J. Hum. Genet. 63, A52.

Giese, K., Cox, K. and Grosschedl, R. (1992). The HMG domain of lymphoid enhancer factor 1 bends DNA and facilitates assembly of functional nucleoprotein structures. Cell 69, 185-195.

Grüneberg, H. (1965). Genes and genotypes affecting the teeth of the mouse. J. Embryol. Exp. Morphol. 14, 137-159.

Grüneberg, H. (1966). The molars of the tabby mouse, and a test of the 'single activated X-chromosome' hypothesis. J. Embryol. Exp. Morphol. 15, 223244.

Grüneberg, H. (1971). The glandular aspect of the tabby syndrome in the mouse. J. Embryol. Exp. Morphol. 25, 1-19.

Headon, D. J. and Overbeek, P. A. (1999). Involvement of a novel TNF receptor homolog in hair follicle development. Nat. Genet. 22, 370-374.

Hoschuetzky, H., Aberle, H. and Kemler, R. (1994). ß-catenin mediates the interaction of the cadherin-catenin complex with epidermal growth factor receptor. J. Cell Biol. 127, 1375-1380.

Jernvall, J., Kettunen, P., Karavanova, I., Martin, L. B. and Thesleff, I. (1994). Evidence for the role of the enamel knot as a control centre in mammalian tooth cusp formation: non dividing cells express growth factor Fgf-4 gene. Int. J. Dev. Biol. 38, 463-469.

Jernvall, J. (1995). Mammalian molar cusp patterns: Developmental mechanisms of diversity. Acta Zool. Fennica 198, 1-61.

Jernvall, J., Åberg, T., Kettunen, P., Keränen, S. and Thesleff, I. (1998). The life history of an embryonic signalling center: BMP4 induces p21 and is associated with apoptosis in the mouse tooth enamel knot. Development 125, 161-169.

Kere, J., Srivasta, A. K., Montonen, O., Zonana, J., Thomas, N., Ferguson, B., Munoz, F., Morgan, D., Clarke, A., Baybayan, P., Chen, E. Y., Ezer, S., Saarialho-Kere, U., de la Chapelle, A. and Schlessinger, D. (1996). $\mathrm{X}$-linked anhidrotic (hypohidrotic) ectodermal dysplasia is caused by a mutation in a novel transmembrane protein. Nat. Genet. 13, 409-416. 
Kratochwil, K., Dull, M., Farinas, I., Galceran, J. and Grosschedl, R. (1996). Lef- 1 expression is activated by BMP-4 and regulates inductive tissue interactions in tooth and hair development. Genes Dev. 10, 13821394.

Mayer, T. C., Miller, C. K. and Green, M. C. (1977). Site of action of the crinkled (cr) locus in the mouse. Dev. Biol. 55, 397-401.

Miard, S., Peterkova, R., Vonesch, J-L., Peterka., M., Ruch., J-V. and Lesot, H. (1999). Alterations in the incisor development in the Tabby mouse. Int. J. Dev. Biol. 43, 517-529.

Micheau, O., Solary, E., Hammann, A. and Dimanche-Boitrel, M. T. (1999). FasL independent, FADD-mediated activation of the Fas death pathway by anti-cancer drugs. J. Biol. Chem. 274, 7987-7992.

Mikkola, M. L., Pista, J., Pekkanen, M., Kere, J. and Thesleff, I. (1998). Characterisation of the mouse Tabby protein, mutation of which causes ectodermal dysplasia. Mol. Biol. Cell 9, 1789.

Mikkola, M. L., Pista, J., Pekkanen, M., Paulin, L., Nieminen, P., Kere, J. and Thesleff, I. (1999). Ectodysplasin, a protein required for epithelial morphogensis, is a novel TNF homologue and promotes cell-matrix adhesion. Mech Dev. 88, 133-146.

Montonen, O., Ezer, S., Saarialho-Kere, U. K., Herva, R., KarjalainenLinsberg, M., Kaitila, I., Sclessinger, D., Srivastava, A. K., Thesleff, I. and Kere, J. (1998). The gene defective in anhidrotic ectodermal dysplasia is expressed in developing epithelium, neuroectoderm, thymus and bone. $J$. Histochem. Cytochem. 46, 281-289.

Monreal, A. W., Zonana, J. and Ferguson, B. (1998). Identification of a new splice form of the EDA1 gene permits detection of nearly all X-linked hypohidrotic ectodermal dysplasia mutations. Am. J. Hum. Genet. 63, 380389.

Monreal, A. W., Ferguson, B. M., Headon, D. J., Street, S. L., Overbeek, P. A. and Zonana, J. (1999). Mutations in the human homolog of the mouse $d l$ cause autosomal recessive and dominant hypohidrotic ectodermal dysplasia. Nat. Genet. 22, 366-369.

Newton, K., Harris, A. W., Bath, M. L., Smith, K. G. C. and Strasser, A. (1998). A dominant interfering mutant of FADD/MORT1 enhances deletion of autoreactive thymocytes and inhibits proliferation of mature $\mathrm{T}$ lymphocytes. EMBO J. 17, 706-718.

Philips, R. J. S. (1960) Mouse News Letter 23, 29.

Pispa, J., Jung, H-S., Jernvall, J., Kettunen, P., Mustonen, T., Tabata, M. J., Kere, J. and Thesleff, I. (1999). Cusp patterning defect in Tabby mouse teeth and its partial rescue by FGF. Dev. Biol. 216, 521-534.

Sarkar, L. and Sharpe, P. T. (1999). Expression of Wnt signalling pathway genes during tooth development. Mech. Dev. 85, 197-200.

Saxén, L. (1966). The effect of Tetracyclin on osteogenesis in vitro. J. Exp. Zool. 162, 269-294.

Schneider, P., MacKay, F., Steiner,V., Hofmann, K., Bodmer, J. L., Holler,
N., Ambrose, C., Lawton, P., Bixler, S., AchaOrbea, H. et al. (1999). BAFF, a novel ligand of the TNF family stimulates B cell growth. J. Exp. Med. 189, 1747-1756.

Schneider, P. (2000). Production of recombinant TRAIL and TRAIL receptors: Fc chimeric proteins. Meth. Enzymol. 322, 325-345.

Sofaer, J. A. (1969a). Aspects of the tabby-crinkled-downless syndrome I. The development of tabby teeth. J. Embryol. Exp. Morphol. 22, 181-205.

Sofaer, J. A. (1969b). Aspects of the tabby-crinkled-downless syndrome II. Observations on the reaction to changes of genetic background. J. Embryol. Exp. Morphol. 22, 207-227.

Sofaer, J. A. (1977). Short communication: The teeth of the 'Sleek' mouse. Arch. Oral Biol. 22, 299-301.

Srivastava, A. K., Pispa, J., Hartung, A. J., Du, Y., Ezer, S., Jenks, T., Shimada, T., Pekkanen, M., Mikkola, M. L., Ko, M. S. H. et al. (1997). The Tabby phenotype is caused by mutations in a mouse homoloue of the EDA gene that reveals novel mouse and human exons and encodes a proetin (ectodysplasin-A) with collagenous domains. Proc. Natl. Acad. Sci. USA 94, 13069-13074.

Sundberg, J. P. (1994). The Downless (dl) and Sleek (Dl Sleek) mutations, Chromosome 10. In Handbook of Mouse Mutations with Skin and Hair Abnormalities (ed. Maibach.H.I.), pp. 241-229. Boca Raton, FL: CRC Press.

Trowel, O. A. (1959). The culture of mature organs in a synthetic medium. Exp. Cell Res. 16, 118-147.

Tucker, A. S., Al Khamis, A., Ferguson, C. A., Bach, I., Rosenfeld, M. G. and Sharpe, P. T. (1999). Conserved regulation of mesenchymal gene expression by Fgf- 8 in face and limb development. Development 126, 221228.

Tucker, A. S. and Sharpe, P. T. (1999). Molecular genetics of tooth morphogenesis and patterning: the right shape in the right place. J. Dental Res. 78, 826-834.

Vaahtokari, A., Åberg., T. and Thesleff. I. (1996a). Apoptosis in the developing tooth: association with an embryonic signalling centre and suppression by EGF and FGF-4. Development 122, 121-129.

Vaahtokari, A., Åberg., T., Jernvall, J., Keränen, S. and Thesleff. I. (1996b). The enamel knot as a signalling centre in the developing mouse tooth. Mech. Dev. 54, 39-43.

van Genderen, C., Okamura, R. M., Farinas, I., Quo, R., Parslow, T. G., Bruhn, L. and Grosschedl, R. (1994). Development of several organs that require inductive epithelial-mesenchymal interactions is impaired in $L E F-1$ deficient mice. Genes Dev. 8, 2691-2703.

Wilkinson, D. G. (1995). In situ hybridisation: a practical approach. Oxford: Oxford University Press.

Zhou, P., Byrne, C., Jacobs, J. and Fuchs, E. (1995) Lymphoid enhancer factor 1 directs hair follicle patterning and epithelial cell fate. Genes Dev. 9, 700-713. 